\title{
Exposure-adjusted racial/ethnic disparities in mortality in the U.S.
}

\section{Arolas, Héctor Pifarré i}

Max Planck Institute for Demographic Research

2021

Arolas , H P I , Acosta , E , Dudel , C , Hale , J M \& Myrskylä , M 2021 ' Exposure-adjusted racial/ethnic disparities in mortality in the U.S. ' MPIDR Working Paper, no. WP-2021-023, Max Planck Institute for Demographic Research , Rostock . https://doi.org/10.4054/MPIDR-WP-2021-023

http://hdl.handle.net/10138/340364

https://doi.org/10.4054/MPIDR-WP-2021-023

publishedVersion

Downloaded from Helda, University of Helsinki institutional repository.

This is an electronic reprint of the original article.

This reprint may differ from the original in pagination and typographic detail.

Please cite the original version. 



\section{Exposure-adjusted racial/ethnic disparities in mortality in the U.S.}

Héctor Pifarré i Arolas, ${ }^{1, *}$ Enrique Acosta, ${ }^{2}$ Christian Dudel, ${ }^{2}$ Jo Mhairi Hale ${ }^{3}$ and Mikko Myrskylä ${ }^{2,4}$

${ }^{1}$ Centre for Research in Health Economics, Universitat Pompeu Fabra, Barcelona, Spain, ${ }^{2}$ Max Planck Institute for

Demographic Research, Rostock, Germany, ${ }^{3}$ School of Geography and Sustainable Development, University of St Andrews, St Andrews, Scotland, ${ }^{4}$ Center for Social Data Science, University of Helsinki, Helsinki, Finland

*Corresponding author. Centre for Research in Health Economics, Universitat Pompeu Fabra, 08002 Barcelona, Spain. E-mail: $\underline{\text { hector.pifarre@upf.edu }}$ 


\section{Abstract}

Background: Racial mortality disparities in the U.S. are well-documented and central to the debate on social inequalities in health. We argue that standard measures that are used to describe the disparities, such as life expectancy or years of life lost, underestimate those disparities.

Methods: We analyze contemporary U.S. mortality disparities comparing Blacks and Hispanics to Whites using CDC and NCHS data. We estimate mortality disparities using standard metrics and a novel approach that weights mortality inequalities by the population fraction that is exposed to the inequalities. We then express the magnitude of these inequalities by comparing them to the loss of life due to leading causes of death.

Results: Based on the exposure-adjusted measure, the Black mortality disadvantage is as deadly or deadlier than circulatory diseases, the top cause of death in the U.S; and $43 \%$ (men) and $87 \%$ (women) larger than the disadvantage as measured by life expectancy. For Hispanics, the exposure-adjusted mortality advantage over Whites is over two times larger, for both men and women, than what life expectancy disparities would imply, and $21 \%$ (men) and 11\% (women) larger than when measured using standard years of life lost.

Conclusions: Mortality inequalities experienced by real populations can differ markedly from the inequalities that are calculated for synthetic populations that are used in standard calculations. We show that racial/ethnic disparities in the U.S. are underestimated if not adjusted for the populations experiencing the inequalities. For health policy the exposure-adjusted inequalities are likely to provide a more reasonable signal on where to allocate scarce resources.

\section{Key messages ( 3 points)}

- Racial mortality inequalities in the U.S. remain large and are a central component in racial inequalities in well-being.

- Commonly used approaches to measure the racial mortality inequalities are based on synthetic (artificial) populations.

- We propose a new exposure-adjusted measure that shows that the disparities experienced by the real population are markedly larger than what standard measures suggest.

Key words: racial disparities, mortality, age structure, exposure

Word count: 3094. 


\section{Introduction}

Racial/ethnic disparities in mortality in the U.S. are large and persistent, despite the recent narrowing of the gap between Black and White Americans. ${ }^{1}$ The standard indicators for measuring and monitoring these inequalities include life expectancy and years of life lost. By design, these indicators are based on synthetic or standard populations; that is, they disregard the actual population age structures when comparing mortality experiences across populations. This feature may be highly beneficial in some contexts, such as when there are differences in population age structures, as with Blacks, Whites, and Hispanics in the U.S. (Whites are older). ${ }^{2}$ For health policy, however, measures that account for population size and structure may be helpful in providing a signal on where to allocate scarce resources.

Furthermore, the age structure-independence property can result in misleading conclusions in some contexts. The current COVID-19 pandemic provides a salient example. The risk of complications and death after infection increases exponentially with age. ${ }^{3,4}$ Therefore, we would expect countries with older populations to be more heavily afflicted by the pandemic. ${ }^{5}$ However, an assessment based on life expectancy would conclude that for two populations the impact of the pandemic would be the same given similar baseline age-specific mortality rates and COVID-19 related age-specific mortality rates, even if one of the populations is relatively older and would thus be more affected. That is, the decline in life expectancy would be the same despite one of the populations experiencing more deaths at old ages. This shortcoming is known, ${ }^{6}$ but often not sufficiently acknowledged.

A key alternative summary measure to life expectancy is years of life lost, which is commonly used to assess the relative importance of specific causes of death within a population and thus guide public health interventions. ${ }^{7}$ Years of life lost are the sum of the years between the age at which death occurs and the age at which we would expect the death to occur, that is, remaining life expectancy. ${ }^{8,9}$ Years of life lost measures need to be directly age-standardized for cross-population comparisons, using a shared population age structure. This is not without controversy, as there is no generally agreed-upon objective way to choose the standard population, and results may vary strongly depending on the standard, ${ }^{10}$ affecting not only the magnitude of disparities but even their direction. ${ }^{11}$ Given the importance of age structures in mortality assessments, this is a partial solution at best. 
We propose a novel measure for evaluating mortality inequalities that accounts for the actual age structures of the populations. In contrast to the common approach used in years of life lost calculations, our proposed exposure-adjusted indicator addresses shortcomings of subjective and direct age-standardization by using the real population exposures. We demonstrate this exposure-adjusted measure with a case study evaluating contemporary mortality inequalities in the U.S. between non-Hispanic Blacks, non-Hispanic Whites, and Hispanics. To highlight the magnitude of the differences among measures, we compare our measure of exposure-adjusted inequalities to the inequalities estimated when using life expectancy and standardized years of life lost. Our findings suggest that standard demographic indicators underestimate the mortality inequalities because they disregard actual population age structures when comparing mortality experiences across populations. The results based on the novel exposure-adjusted indicator, which we call the exposure-adjusted mortality gap, suggest that inequalities are substantially larger than standard demographic methods would imply.

\section{Methods}

\section{The measurement of the racial mortality gap}

The object of interest of this study, the racial mortality gap, is measured as the difference between given mortality indices across two races $(A, B)$. In the case of comparisons based on life expectancy, this is simply given by:

$$
\Delta e_{0}^{A, B}=e_{0}^{A}-e_{0}^{B}(1)
$$

where $e_{0}^{G}$ stands for the life expectancy at birth of racial group $G$; for simplicity, we denote the mortality gap measured using this approach as $\Delta e_{0}$. Another common strategy is based on the years of life lost (YLL) framework. ${ }^{8,9}$ While typically YLL are used to assess cause-related mortality, they can also be utilized to assess the racial mortality gap. The standard approach calculates YLL per death as the difference between the age at death and the life expectancy at that given age. At a population level, they are often expressed in rates (per 100k); for population $G$, YLL (rates) are given by:

$$
Y L L_{G}=\sum_{x} m_{x}^{G} \cdot e_{x} \cdot C_{x}^{G} \cdot 100000
$$


where $m_{x}^{G}, e_{x}, C_{x}^{G}$ are the age-specific mortality rates, standard remaining life expectancy for persons dying in each age group, and fraction of the population, respectively. In cross-population comparisons, such as racial/ethnic groups, they are typically (directly) age-standardized employing a reference standard population $\left(C_{x}^{s}\right)$, which we denote $s Y L L$. This version of the racial mortality gap, denoted $\triangle s Y L L$, is thus:

$$
\triangle S Y L L_{A, B}=s Y L L_{A}-s Y L L_{B}
$$

\section{A counterfactual approach to racial mortality inequality}

We propose a counterfactual method to the measurement of mortality disparities. The basic idea, in the spirit of indirect standardization methods, consists of measuring the disparity between a population A and its reference B as the mortality change for A from attaining B's mortality rates while holding constant A's age structure. We embed this calculation in the standard years of life lost (YLL) approach. We call this indicator the exposure-adjusted mortality gap $(e \Delta Y L L)$.

We operationalize this idea in the following manner. Instead of using direct age-standardized YLL $(s Y L L)$, we compute a counterfactual YLL $(c Y L L)$, with A's age structure and B's mortality rates.

$$
c Y L L_{A}=\sum_{x} m_{x}^{B} \cdot e_{x} \cdot C_{x}^{A} \cdot 100000
$$

Then, we measure the MG as the difference between $Y L L^{c}$ and the actual YLL, i.e. the YLL at both A's current mortality rates and population age structure; that is, $e \Delta Y L L$ is the difference between (2) and (4).

$$
e \Delta Y L L_{A, B}=Y L L_{A}-c Y L L_{A}(5)
$$

Thus, a positive $e \Delta Y L L(5)$ shows how many years of life are lost (a "disadvantage") and a negative $e \Delta Y L L$ how many years are gained (an "advantage") relative to a reference population's mortality. This age-structure-dependent measure of mortality differences can then be used as the foundation to study the racial/ethnic mortality disparity, without arbitrarily choosing a population for (direct) age standardization.

Similar counterfactual approaches are frequently used in demography, epidemiology, and public health. Our proposal follows the logic of indirect standardization methods. ${ }^{12}$ Indirect standardization measures, such as the 
standardized mortality ratio, compare observed death counts to a counterfactual based on the actual population age structure and the reference population's mortality rates. We embrace this approach and extend it to consider the age gradient of the loss of life accrued by deaths. How can these counterfactual mortality rates be understood? Mortality disparities are rooted in mutable social inequities, such as socioeconomic disparities and racial discrimination. For example, the approximately double infant mortality rates Blacks suffer compared with Whites ${ }^{13}$ have been tied to access to care, ${ }^{14}$ race-related biases at treatment, ${ }^{15}$ and other social factors. ${ }^{16}$ Thus, it is conceivable that disadvantaged racial/ethnic groups might achieve the lower mortality rates of the more advantaged. This is our counterfactual.

Intuitively, population groups may place more importance on mortality risks affecting larger fractions of the population. In the infant mortality rate example above, for a group with a young population with high fertility rates, reducing infant mortality could represent a greater reduction in loss of life than improving mortality rates at the upper end of the mortality distribution. And the contrary could be true for a relatively older population group, as in the COVID-19 example above, where older populations may focus more resources on COVID-19 prevention. Thus, adjusting for the actual age structure of the population may lead to a greater understanding of how to prioritize public health interventions to reduce loss of life. Our measure, $e \Delta Y L L$, allows us to evaluate these trade-offs.

\section{Comparisons to other approaches}

In order to compare our results to those of existing methods measuring the mortality gap using life expectancy $\left(\Delta e_{0}\right)$ or standardized YLL $(\Delta s Y L L)$, we normalize each measure by dividing it by the loss of life from leading causes of death. This normalization serves two purposes. First, it serves as a reference for the magnitude of the $e \Delta Y L L,{ }^{2}$ by comparing it with important causes of death (COD) with which we are already familiar. Second, it facilitates the comparison of the racial gap across metrics using different units of measure. The importance of these causes of death within a racial/ethnic group is assessed using standard approaches; for $e_{0}$, we use a cause-deleted life table approach ${ }^{11}$ and for YLL, we compute the YLL associated with each COD. ${ }^{8,9}$

We measure racial mortality gaps through counterfactuals based on examining actual populations with alternative mortality rates. In contrast, both $\Delta e_{0}$ and $\Delta s Y L L$ evaluate the racial mortality disparities for 
counterfactuals based on synthetic populations with actual mortality rates. In the case of $\Delta s Y L L$, it captures the racial mortality inequalities that would exist should all races share the age structure of a reference population (e.g., the U.S. standard population). In turn, $\Delta e_{0}$ based assessments can be given a population interpretation, whereby differences in life expectancy at birth between race-specific populations are the disparity in mean ages at death in their respective stationary populations (stable -- long run -- populations based on current mortality rates and no population growth). Therefore, both approaches evaluate mortality disparities in synthetic / theoretical populations that do not reflect the fraction of the populations suffering from the existing mortality disparities. Figure 1 illustrates the differences in age structure by race and gender between the current population and the life table and the U.S. standard populations. Both the Hispanic and Black actual populations are younger than the life table and standard populations suggest (more in the Hispanic case), whereas the reverse is true for Whites.

\section{Application}

We use the $e \Delta Y L L$ to re-analyze contemporary racial/ethnic mortality inequalities in the U.S., using the National Center for Health Statistics (NCHS) data. We compare non-Hispanic Blacks and Hispanics (irrespective of race) to non-Hispanic Whites. We use circulatory diseases, the leading cause of death in the U.S., for normalizing racial disparities. Table 1 displays all the elements of these calculations and the results. We divide the racial mortality gap calculated according to each approach (table 1, column 4) by the loss of life from a cause of death (table 1, column 5). For Blacks, the normalized mortality disparity is interpreted as "the mortality disadvantage of Blacks compared to Whites is X times as much loss of life as circulatory diseases." Hispanics have lower mortality than Whites, so the ratio captures the mortality advantage of Hispanics. We calculate similar relative metrics based on $\Delta e_{0}$ and $\Delta s Y L L$

\section{Data}

Life tables for year 2018 by single year of age, sex, race, and Hispanic origin were retrieved from the NCHS Life Tables website's public files. ${ }^{17}$ Death counts for 2018 by cause, single-year of age, sex, race, and Hispanic origin were obtained from the Mortality Multiple Cause Files, available through the Vital Statistics online data portal. ${ }^{18}$ Causes of death were defined following the 10th revision of the International Statistical Classification 
of Diseases (ICD-10). The U.S. standard population and U.S. population estimates by single year of age $(0,1,2, \ldots$, 85 years and over), abridged race, Hispanic origin, and sex, on July 1, 2018, were taken from the abridged-race intercensal estimates of the resident population of the United States website. ${ }^{19}$ Standard life expectancies by single year of age and a $85+$ open-ended age interval were taken from the WHO. ${ }^{8}$

\section{Results}

Figure 2 (Panel A) shows the racial/ethnic mortality disparity for Blacks and Hispanics based on life expectancy $\left(\Delta e_{0}\right)$, the direct age standardized (using the U.S. standard population) YLL $(\triangle s Y L L$ ), and the exposure-adjusted racial mortality gap $(e \Delta Y L L)$. Each point represents the ratio of the racial/ethnic disparity to the gains from removing circulatory diseases. We find that, across race/ethnicity and gender, mortality disparities based

on $e \Delta Y L L$ are larger than those captured by $\Delta e_{0}$. The Black/White disparity in $\Delta e_{0}$ is two thirds $(0.66)$ the size of the loss of life expectancy from circulatory diseases for the total Black population. However, based on YLL - both $\triangle S Y L L$ and $e \triangle Y L L$ - the racial disadvantage is as deadly as the leading COD (1.02 and 1.1). That is, the Black/White racial disparity is $67 \%(1.07 / 0.66)$ larger based on our approach. The Hispanic mortality advantage based on $\Delta s Y L L$ and $e \Delta Y L L(1.31$ and 1.5$)$ is more than two times larger than what the $\Delta e_{0}$ based metric implies (0.61). In some cases $\triangle s Y L L$ and $e \triangle Y L L$ differ substantially. For instance, for Hispanic men compared with White men, $\Delta s Y L L$ indicates that ethnic disparities are roughly as deadly as circulatory disease (1.08), while the $e \Delta Y L L$ indicates that ethnic disparities are a considerably bigger killer (1.31).

\section{Gender specific results}

The gender specific results show, across measures, that the mortality disadvantage is higher for Black males than females, and that Hispanic females have a greater mortality advantage than males. However, gender disparities within racial/ethnic groups vary by measure. According to $\Delta e_{0}$, the racial gap is $39 \%(0.53 / 0.87)$ smaller for Black females than males, whereas based on the $e \Delta Y L L$ it is $20 \%(0.99 / 1.24)$ smaller. We find the opposite results for Hispanics, with a larger advantage for females based on $e \Delta Y L L, 35 \%(1.77 / 1.31)$, compared to $\Delta e_{0}, 5 \%(0.61 / 0.58)$. 


\section{Comparison to other leading causes of death}

In figure 2 (Panel B) we normalize by the five leading causes of death in the U.S. to assess whether our results are anomalous to circulatory disease or demonstrative of a pattern. We find that racial/ethnic disparities based on existing approaches $\left(\Delta e_{0}, \Delta s Y L L\right)$ are smaller across almost all causes of death. The exception is external causes, for which the disparity is larger based on $\Delta e_{0}$ than on our approach. This is in part due to the relatively young population composition of Hispanics and Blacks, combined with the fact that, unlike most causes of death, external causes disproportionately affect younger ages. Thus, results for external causes cannot be interpreted as an indication that the racial mortality disparity is relatively smaller according to our counterfactual-based analysis.

\section{Discussion}

Our results show that racial/ethnic mortality evaluations that account for actual population age structures indicate larger mortality disparities than analyses based exclusively on differences in mortality rates, such as life expectancy. Based on our exposure-adjusted measure, the $e \Delta Y L L$, we find a $(67 \%)$ larger Black/White mortality disparity, and a greater (146\%) Hispanic mortality advantage than what life expectancy based calculations would imply. These disparities are also larger than the results obtained using standardized years of life lost measures, indicating that using real age structures instead of standard age structures identifies greater racial/ethnic mortality disparities in the U.S. context.

Our measure complements existing approaches to measure mortality trends and disparities; ultimately, the best approach depends on the question. We have posited that exposures ought to play a central role in understanding population-level disparities, and as such, the $e \triangle Y L L$ is particularly well-suited to study racial/ethnic mortality inequalities. The focus on exposures might not always be warranted. Age-specific life expectancies are commonly used as population-based estimates of remaining life years for actuarial calculations, such as the ones involved in the forecasts of pension expenditures. ${ }^{20}$ In trying to understand and model individual behaviour (e.g. savings decisions), individual survival probabilities play a central role. ${ }^{21}$ Finally, we might want to hold age structures constant in assessing temporal trends in issues such as the burden of disease, as to assess the improvement of condition-specific 
mortality rates. ${ }^{22}$ Our approach is thus tailored for analyses in which age-structures are a fundamental component, not merely a confounder.

Similar to existing approaches, a shortcoming of our approach is that we do not consider the effect changing mortality rates could have on age structures. This is clearly the case for $\triangle s Y L L$, since direct age standardization assumes that different mortality rates can coexist with an identical age distribution. It might appear that $\Delta e_{0}$ does not suffer for this shortcoming. Indeed, in the calculation of $e_{0}$, the age structure is implicitly derived from the mortality rates. However, the implicit age structure of the life table is based exclusively on mortality rates, and thus does not represent a realistic approximation of the age structure under alternative mortality distributions.

Beyond technical considerations, the approaches to mortality evaluation we have presented also correspond to distinct perspectives on racial equity in mortality. The underlying notion of equity behind $\Delta e_{0}$-based analysis is that equality will be achieved when any two individuals born in the U.S., regardless of race/ethnicity, have the same life expectancy (individual equality). Alternatively, we have presented a different version whereby equality implies that, given their age structure, no race/ethnicity would be better off exchanging their mortality rates with those of any other race/ethnicity (population equality). The two notions are not equivalent, as we have illustrated in this work, and thus can lead to different recommendations for policies that pursue the reduction of disparities. Given that both approaches have similar data requirements and analytical complexity, the preferred approach will depend on the research question.

\section{Conclusion}

The purpose of this piece is to introduce a new approach to measuring mortality disparities. The exposure-adjusted mortality gap ( $e \Delta Y L L)$ explicitly incorporates age structures in cross-population analyses. We also compare the $e \Delta Y L L$ to the disparity found using two common alternatives - life expectancy and years of life lost. Other indicators that quantify additional dimensions of mortality and health disparities might benefit from insights from this work, such as measures of life table based longevity like the median and modal ages at death ${ }^{23}$ and lifespan inequality indices. ${ }^{24}$ Other measures, such as quality adjusted years of life years (QALY), often used in policy evaluations, ${ }^{25}$ consider the disability status of years lived. While these measures provide additional insights on 
racial/ethnic mortality disparities beyond those covered by our approach, the exposure-related considerations at the core of our contribution also apply. Exploring the quantitative implications of incorporating exposures into these measures represents an interesting potential avenue for future research.

\section{Funding}

EA received funding from the Social Sciences and Humanities Research Council (Canada) - Postdoctoral grant No. 756-2019-0768, and the Fonds de recherche du Québec - Société et culture - Postdoctoral grant No. 274299

\section{Data availability}

All data and code to fully reproduce the analyses are available at the OSF: https://osf.io/hj8g3 .

\section{Author contributions}

HPA and MM contributed to conceptualization, methodology, writing the original manuscript, and review and editing. HPA conducted formal analysis, supervision. EA contributed to methodology, formal analysis, data curation, visualization, and review and editing. CD contributed to methodology, formal analysis, and review and editing. JMH wrote and edited the manuscript.

\section{Conflicts of interest}

None declared.

\section{References}

1. Harper S, MacLehose RF, Kaufman JS. Trends in the black-white life expectancy gap among US states, 1990-2009. Health affairs. 2014 Aug 1;33(8):1375-82.

2. Wrigley-Field E. US racial inequality may be as deadly as COVID-19. Proceedings of the National Academy of Sciences. 2020 Sep 8;117(36):21854-6.

3. Sasson I. Age and COVID-19 mortality: A comparison of Gompertz doubling time across countries and causes of death. Demographic Research. 2021 Feb 17;44:379-96.

4. Goldstein JR, Lee RD. Demographic perspectives on the mortality of COVID-19 and other epidemics. Proceedings of the National Academy of Sciences. 2020 Sep 8;117(36):22035-41.

5. Dudel C, Riffe T, Acosta E, van Raalte A, Strozza C, Myrskylä M. Monitoring trends and differences in COVID-19 case-fatality rates using decomposition methods: Contributions of age structure and age-specific fatality. PLOS one. 2020 Sep 10;15(9):e0238904.

6. Vaupel JW. How change in age-specific mortality affects life expectancy. Population studies. 1986 Mar $1 ; 40(1): 147-57$.

7. Adam T. Making choices in health: WHO guide to cost-effectiveness analysis. World Health Organization; 2003.

8. Martinez R, Soliz P, Caixeta R, Ordunez P. Reflection on modern methods: years of life lost due to premature mortality - a versatile and comprehensive measure for monitoring non-communicable disease mortality. International journal of epidemiology. 2019 Aug 1;48(4):1367-76. 
9. WHO G. WHO methods and data sources for global burden of disease estimates 2000-2011. Geneva: Department of Health Statistics and Information Systems. 2013 Nov.

10. Wyper GM, Grant I, Fletcher E, McCartney G, Fischbacher C, Stockton DL. How do world and European standard populations impact burden of disease studies? A case study of disability-adjusted life years (DALYs) in Scotland. Archives of Public Health. 2020 Dec;78(1):1-8.

11. Preston S, Heuveline P, Guillot M. Demography: measuring and modeling population processes. 2001. Malden, MA: Blackwell Publishers. 2000.

12. Inskip H, Beral V, Fraser P, Haskey J. Methods for age-adjustment of rates. Statistics in medicine. 1983 Oct;2(4):455-66.

13. Driscoll AK, Ely DM. Effects of Changes in Maternal Age Distribution and Maternal Age-specific Infant Mortality Rates on Infant Mortality Trends: United States, 2000-2017. National Vital Statistics Reports: From the Centers for Disease Control and Prevention, National Center for Health Statistics, National Vital Statistics System. 2020 Jun 1;69(5):1-8.

14. Kitsantas P, Gaffney KF. Racial/ethnic Disparities in Infant Mortality. Journal of perinatal medicine. 2010;38(1):87-94.

15. Greenwood BN, Hardeman RR, Huang L, Sojourner A. Physician-patient racial concordance and disparities in birthing mortality for newborns. Proceedings of the National Academy of Sciences. 2020 Sep 1;117(35):21194-200.

16. Geronimus AT. The weathering hypothesis and the health of African-American women and infants: evidence and speculations. Ethnicity \& disease. 1992 Jan 1;2(3):207-21.

17. NCHS, Life tables. https://www.cdc.gov/nchs/products/life tables.htm (15 July 2021, date last accessed).

18. NCHS, Vital statistics online data portal. https://www.cdc.gov/nchs/products/life tables.htm (15 July 2021, date last accessed).

19. NCHS, Abridged-race population estimates. https://www.cdc.gov/nchs/nvss/bridged_race/216data documentation.htm (15 July 2021, date last accessed).

20. Social Security Administration, The 2021 Annual Report of the Board of Trustees of the Federal Old-Age and Survivors Insurance and Federal Disability Insurance Trust Funds, 2021, https://www.ssa.gov/oact/TR/2021/index.html (accessedNovember 15, 2021)

21. Post T, Hanewald K. Longevity risk, subjective survival expectations, and individual saving behavior. Journal of Economic Behavior \& Organization. 2013 Feb 1;86:200-20.

22. Murray CJ, Vos T, Lozano R, Naghavi M, Flaxman AD, Michaud C, Ezzati M, Shibuya K, Salomon JA, Abdalla S, Aboyans V. Disability-adjusted life years (DALYs) for 291 diseases and injuries in 21 regions, 1990-2010: a systematic analysis for the Global Burden of Disease Study 2010. The lancet. 2012 Dec 15;380(9859):2197-223.

23. Canudas-Romo V. Three measures of longevity: Time trends and record values. Demography. 2010 May;47(2):299-312.

24. van Raalte AA, Sasson I, Martikainen P. The case for monitoring life-span inequality. Science. 2018 Nov 30;362(6418):1002-4.

25. Neumann PJ, Cohen JT, Weinstein MC. Updating cost-effectiveness - the curious resilience of the \$50,000-per-QALY threshold. N Engl J Med. 2014 Aug 28;371(9):796-7. 
Tables and figures

\begin{tabular}{|c|c|c|c|c|c|}
\hline Race & Gender & Metric & Mortality gap & $\begin{array}{l}\text { Gain from } \\
\text { circulatory } \\
\text { diseases } \\
\text { elimination }\end{array}$ & $\begin{array}{c}\text { Ratio of } \\
\text { mortality gap } \\
\text { to life loss } \\
\text { circulatory } \\
\text { diseases }\end{array}$ \\
\hline \multirow{9}{*}{ Black } & \multirow{3}{*}{ Female } & $\Delta e_{0}$ & 3.11 & 5.92 & 0.53 \\
\hline & & $\Delta s Y L L$ & 4841.49 & 5003.87 & 0.97 \\
\hline & & $e \Delta Y L L$ & 5118.75 & 5171.75 & 0.99 \\
\hline & \multirow{3}{*}{ Male } & $\Delta e_{0}$ & 4.91 & 5.65 & 0.87 \\
\hline & & $\Delta s Y L L$ & 9301.01 & 8487.66 & 1.10 \\
\hline & & $e \Delta Y L L$ & 9259.43 & 7448.90 & 1.24 \\
\hline & \multirow{3}{*}{ Total } & $\Delta e_{0}$ & 3.92 & 5.93 & 0.66 \\
\hline & & $\Delta s Y L L$ & 6688.90 & 6547.06 & 1.02 \\
\hline & & $e \Delta Y L L$ & 6913.89 & 6267.33 & 1.10 \\
\hline \multirow{9}{*}{ Hispanic } & \multirow{3}{*}{ Female } & $\Delta e_{0}$ & -3.22 & 5.31 & 0.61 \\
\hline & & $\Delta s Y L L$ & -3632.78 & 2266.27 & 1.60 \\
\hline & & $e \Delta Y L L$ & -2937.75 & 1655.30 & 1.77 \\
\hline & \multirow{3}{*}{ Male } & $\Delta e_{0}$ & -2.88 & 4.99 & 0.58 \\
\hline & & $\Delta s Y L L$ & -4352.55 & 4039.22 & 1.08 \\
\hline & & $e \Delta Y L L$ & -3341.36 & 2551.48 & 1.31 \\
\hline & \multirow{3}{*}{ Total } & $\Delta e_{0}$ & -3.20 & 5.28 & 0.61 \\
\hline & & $\Delta s Y L L$ & -4045.20 & 3076.66 & 1.31 \\
\hline & & $e \Delta Y L L$ & -3159.35 & 2100.87 & 1.50 \\
\hline
\end{tabular}

Table 1. The table presents $\Delta e_{0}, \Delta s Y L L, e \Delta Y L L$ for: the racial gap between each race and Whites (column 4), the loss of life from circulatory diseases for each race (column 5), and the ratio of the two (column 6). In the case of Hispanics, the mortality gap is negative (advantage), whereas for Blacks it is positive (disadvantage). 


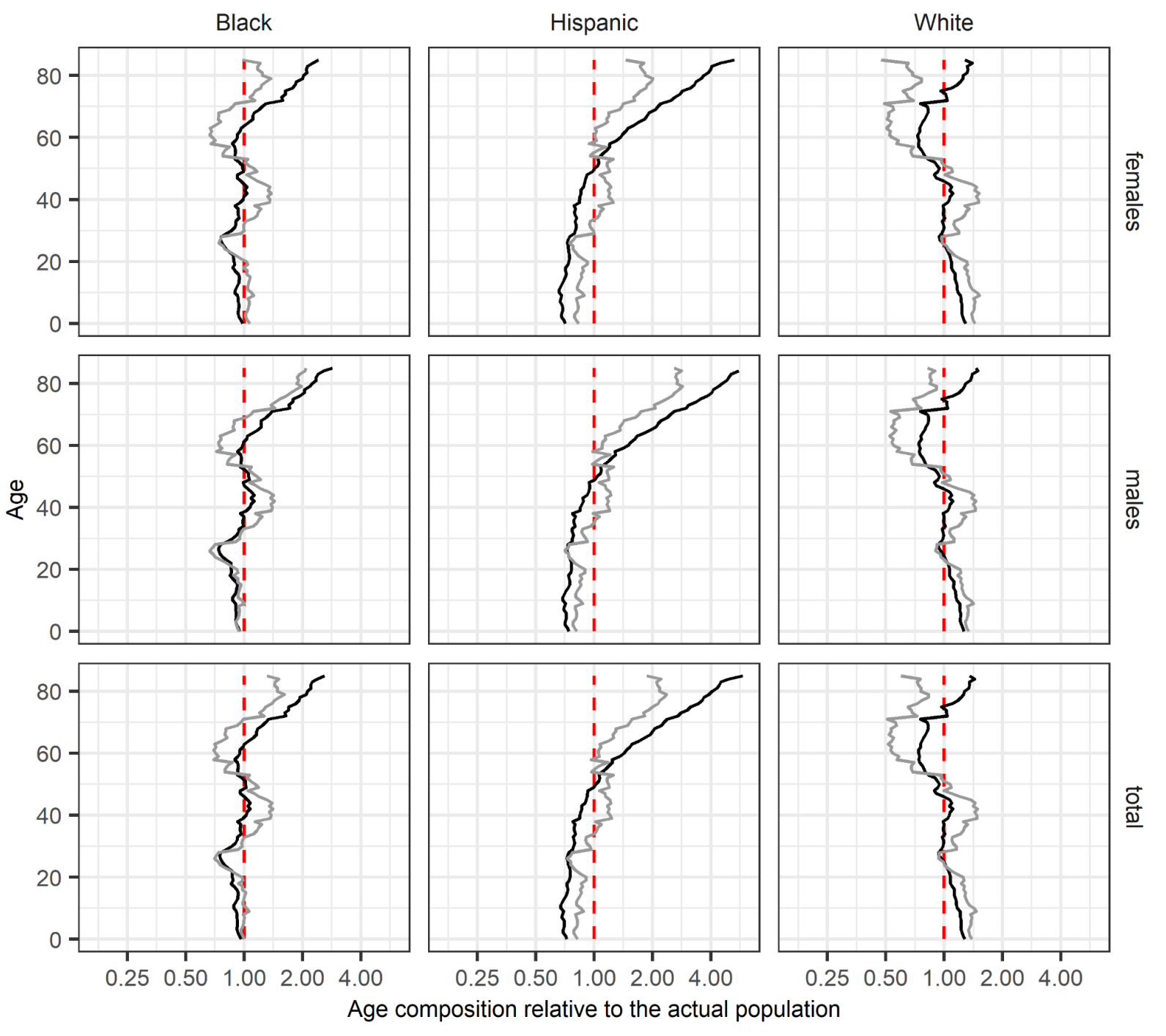

Population - - Actual — US Standard — Life Table

Figure 1. Deviations from the actual age distribution (2018). 
A
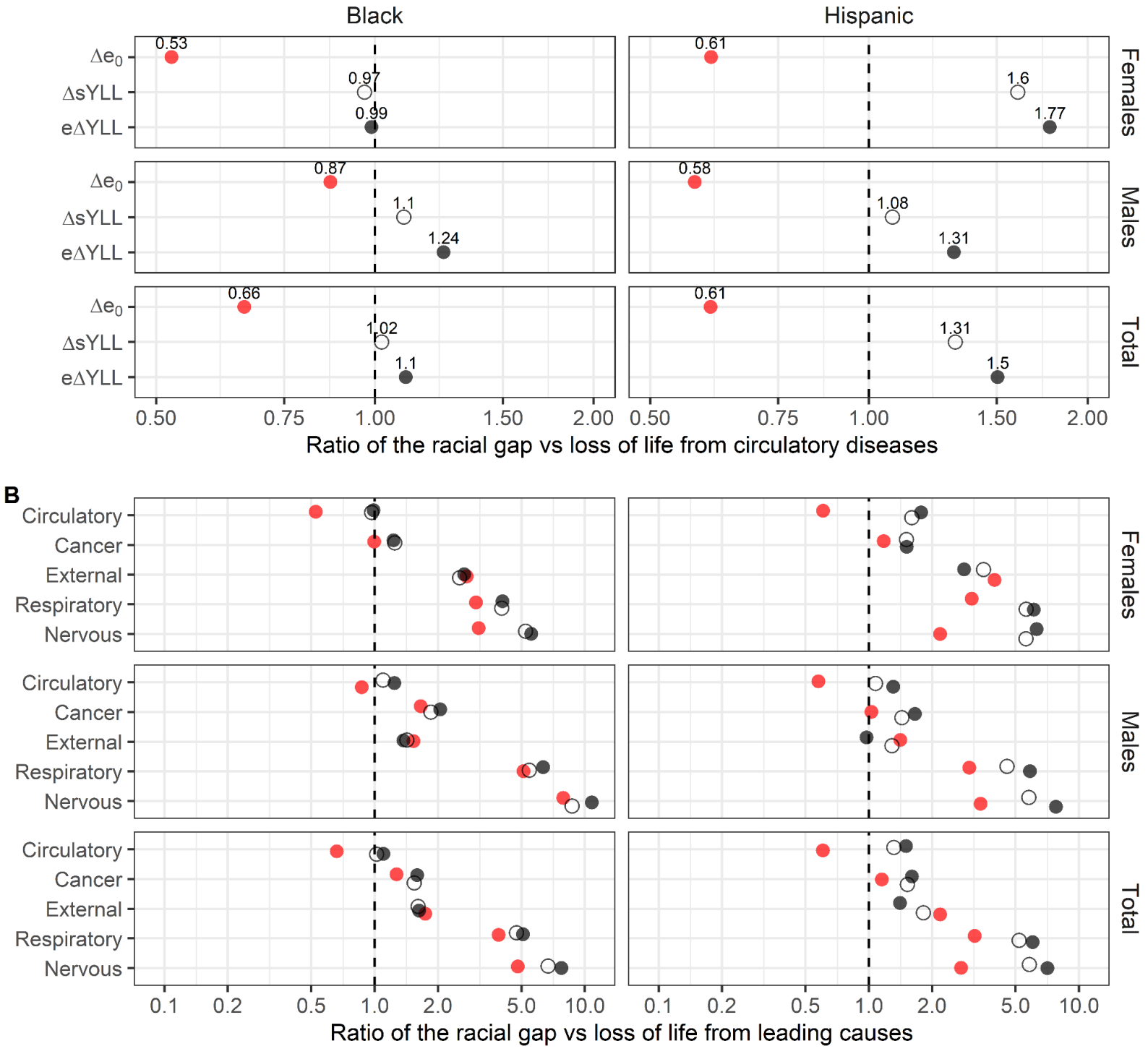

Measure $\bullet \Delta \mathrm{e}_{0} \bigcirc{ }_{\Delta s \mathrm{YLL}} \bullet \mathrm{e} \Delta \mathrm{YLL}$

Figure 2. Mortality gap based on life expectancy $\left(\Delta e_{0}\right)$, standardized YLL $(\Delta s Y L L)$ and exposure-adjusted YLL ( $e \Delta Y L L)$. Panel A displays the racial/ethnic disparity for females, males, and total in relation to circulatory diseases. Panel B reports the racial/ethnic disparity for females, males, and total using other causes of death (top 5 causes) as a reference. 